\title{
Effect of test- and -treat strategy on antiretroviral drugs uptake in a prevention of mother to child transmission programme in Southwest Nigeria
}

\author{
Abayomi Joseph Afe ${ }^{1}$, Isah Haroun ${ }^{2}$, Kemi Edet-Utan ${ }^{1}$, Timothy Akinmurele ${ }^{1}$ \\ ${ }^{1}$ Community Medicine Department, Equitable Health Access Initiative, Lagos Nigeria \\ ${ }^{2}$ Community Health Department, Institute of Human Virology Nigeria, Abuja, Nigeria \\ Email address: \\ abayomiafe@yahoo.com (A. J. Afe)
}

\section{To cite this article:}

Abayomi Joseph Afe, Isah Haroun, Kemi Edet-Utan, Timothy Akinmurele. Effect of Test- and -Treat Strategy on Antiretroviral Drugs Uptake in a Prevention of Mother to Child Transmission Programme in Southwest Nigeria. Science Journal of Public Health.

Vol. 2, No. 5, 2014, pp. 476-479. doi: 10.11648/j.sjph.20140205.25

\begin{abstract}
A multicenter retrospective case-control study. Study subjects were HIV positive pregnant women enrolled in a prevention of mother to child transmission of HIV(PMTCT) programme in southwest Nigeria. The objective was to see if the introduction of 'Test- and -Treat strategy' would lead to placing higher number of HIV-POSITIVE pregnant women on antiretroviral drugs. There was a significant difference $(\mathrm{OR}=1.4 ; \mathrm{CI}=1.35-1.55, \mathrm{P}=0.0)$ in the proportions of HIV-POSITIVE pregnant women initiated on ARV between the control $\operatorname{arm}(60 \%)$ and the case group $(81 \%)$ with the implementation of the strategy. In other words, an HIV POSITIVE woman in a PMTCT setting has a $40 \%$ greater chance of being placed on ARV with test-and-treat strategy than without the strategy.The test- and -treat strategy is a treatment approach in which HIV positive clients are initiated on prophylactic antiretroviral drugs immediately after HIV diagnosis without waiting for the CD4 count test result. Hence, implementing Test and Treat Strategy in a PMTCT programme increases the antiretroviral drug uptake by HIV-POSITIVE women which in turn could lead to a reduced rate of vertical transmission of HIV, as a result of the suppressed maternal viral load.
\end{abstract}

Keywords: PMTCT (Prevention of Mother To Child Transmission of HIV), IHVN (Institute of Human Virology Nigeria), CD4-T-lymphocytes (CD4 Cells), ART (Antiretroviral Therapy), PLHIV (People Living with HIV), Antiretroviral Drugs (ARV)

\section{Objective}

To demonstrate increase in the uptake of antiretroviral drugs among PMTCT clients using the test and treat strategy

\section{Research Question}

Use Of Test And Treat Strategy Increases The Proportion Of HIV Positive Pregnant Women Placed On Antiretroviral Drugs For PMTCT

\section{Introduction}

In 2012, an estimated 35.3 million (32.2 million - 38.8 million) people globally were living with HIV, out of which 2.3 million (1.9 million - 2.7 million) people were newly infected with HIV in 2012 alone and 1.6 million (1.4 million - 1.9 million) people died from AIDS-related illnesses in the same year (1). Even though antiretroviral treatment (ART) has emerged as an effective tool for saving lives of people living with HIV (PLHIV), yet in 2011 coverage of effective ARV drug regimens for PMTCT was 57\% in low- and middle-income countries. However in the same year only $30 \%$ of HIV positive pregnant women who needed ART for their own health received it compared with 54\% ART coverage for all eligible adults in low- and middle-income countries(2). Reasons for this include lack of ARV supply especially to PLHIV, who are in hard-to-reach rural settings in subSaharan nations like Nigeria. The requirement for a compulsory baseline CD4 count test as stipulated in most old treatment guidelines also accounted for the low ARV uptake by HIV positive pregnant women. This is more so in settings where these drugs are available but the tests are not readily 
accessible. In many health facilities where ARV for prevention of mother to child transmission of HIV (PMTCT) services are being offered, only a few can boast of functional CD4 equipment and still fewer can provide same day results that are needed for rapid assessment of the clients. The majority of these health centers pulls blood samples from patients and transports these to the nearest laboratories for analysis. With this arrangement, not all the patients get their results as some may be lost in transit and patients may even fail to turn up again for their results. The testing laboratory too may disqualify some of these blood samples with the attendant inability to recall those patients for repeat blood collection. All these factors have combined to make the rate of ARV uptake among PMTCT clients scarcely above average in Nigerian setting thereby impacting negatively on reduction of vertical HIV transmission .Theoretically with the early and immediate initiation of ARV in PLHIV using the test and treat strategy, more patients will be placed on drugs as those who would have been disqualified from ARV initiation because of their CD4 test result will be initiated on drugs. Most earlier studies on test and treat strategy in HIV were done among serodiscordant couples. The objective of which was to use it as a prevention strategy to reduce sexual HIV transmission to the seronegative partners by reducing community viral load (3).No local study on test and test strategy among PMTCT clients is known to the author, hence this study.

Nigeria had a national PMTCT coverage of $17 \%$ as at 2012(4) and contributed 30\% to the world gap in achieving a global target of eradicating MTCT, making her the highest contributor to paediatric HIV infection in the world(5). The number of HIV-INFECTED pregnant women in 2011 was 221,129 with only $17.1 \%$ receiving antiretroviral to reduce MTCT. It is estimated that $26.5 \%$ $(58,495)$ of the HIV-INFECTED pregnancies led to child infections in the same year (6).

\subsection{Test and Treat Protocol}

The test-and-treat strategy involves an aggressive expansion of HIV testing to identify HIV INFECTED persons, and immediate initiation of antiretroviral therapy. For those diagnosed HIV positive, they are placed on ARV without waiting for the results of laboratory investigations such as the CD4 count i.e. patients are started on $A R V$ drugs irrespective of their CD4 count result as stipulated by the 2013 WHO PMTCT guideline. Though blood samples for CD4 count and other baseline tests like the liver function tests, Hepatitis B and C tests, full blood count, electrolytes, serum urea and creatinine are still drawn immediately after the post-test counseling, the physicians go ahead to prescribe the ARV adopting either Option B or Option B+ ARV regimen(7). Adherence counselling is also initiated at the same time. Substitution and switching of the appropriate ARV drug can, of course, still occur based on the outcome of the baseline laboratory tests later in the course of management(7).For instance, Zidovudine can be substituted with Abacavir if the packed cell volume( PCV) comes out less than $24 \%$, Tenofovir can be substituted in patients with abnormal renal function test. To avoid the hepatotoxicity associated with Nevirapine at CD4 count of above 250 cells $/ \mathrm{mm} 3$ in women Nevirapine is substituted with Efavirenz in Test and Treat strategy for HIV POSITIVE pregnant women. In fact, a fixed-dose formulation of Tenofovir (TDF) + Lamivudine or Emtricitabine (3TCor FTC) + Efavirenz (EFZ )which is taken once daily and, therefore, facilitate excellent adherence makes a good regimen for test and treat (10). Starting ARV in $\mathrm{HIV}+$ pregnant women without waiting for baseline CD4 tests results as part of the test and treat approach allows for early initiation of ARV. This in turn leads to longer duration on ARV before delivery. Thus increases the likelihood of maternal viral load suppression at delivery and, therefore, decreases vertical transmission of HIV(11). Other advantages of Test and Treat HIV treatment include increased ease of ART initiation, fast ART initiation process, tendency to place more patients on ARV compatible with the Nigerian national scale -up plan objective of placing at least $90 \%$ of HIV positive pregnant women and breastfeeding mother-infant pair on ARV by 2015. Also it is a strategy that can easily be implemented at the lower levels of healthcare service like the primary and secondary health facilities. This is especially important in low resource setting like Nigeria where out of a total of 22,726 health facilities in Nigeria, 94\% of these facilities are primary health care facilities, where majority of Nigerians access healthcare services(12). Involvement of this lower cadre of healthcare services will help in the accelerated scale up towards elimination of Mother to Child transmission of HIV(eMTCT) through integration and decentralization of PMTCT services from tertiary and secondary facilities to primary care facilities Achievement of the Nigerian elimination of Mother to Child transmission of HIV(eMTCT) target will definitely have a positive impact on the attainment of the world Millenium Development Goals 4 and 5.On the other hand,the testand-treat strategy could also lead to indiscriminate use of ARV, poor adherence which may lead to emergence of resistant HIV strains, especially if adherence counselling is poorly done.Supply of ARV for the large population of PLHIV could also be a challenge in test and treat, especially in low resource settings where foreign donors account for more than $90 \%$ of the HIV consumables including ARV. Despite this, test-and-treat strategy is a good public health approach in HIV management that could be used to implement the use ARV for prevention, which has the potential of reducing community viral load and significantly decrease HIV transmission in the community.

\section{Methodology}

The research is a retrospective case-control study comparing the proportions of HIV-POSITIVE pregnant women who had antiretroviral drugs for PMTCT among the cases with the controls. The control comprised of all HIV 
positive pregnant women $(\mathrm{N}=28,918)$ seen between January 2005 and September 2011 when CD4 count test result was a pre-requisite to commencement of ARV in PMTCT across the 200 PEPFAR-supported study sites spread across Nigeria. While the cases were the HIVPOSITIVE pregnant women $(\mathrm{N}=1,779)$ seen at 64 health facilities in southwest Nigeria from October 2011 to October 2013 where the 'Test-and Treat' strategy was implemented. Study period was from January 2005 to Sept.2013 across 200 control sites and 64 case sites. These were all IHVN-ACTION PEPFAR supported health facilities spread across Nigeria. The study population was the HIV-POSITIVE pregnant women and the only criterion for their inclusion was enrolment at the study centers. Study data were the routine program data captured with the national monitoring and evaluation tools developed for the PEPFAR project in Nigeria

\section{Results}

Table 1. Prevalence Of Hiv Infection Among Anc Clients

\begin{tabular}{llll}
\hline \multirow{2}{*}{ DATES } & $\begin{array}{l}\text { Had HCT } \\
\text { Done }\end{array}$ & $\begin{array}{l}\text { HIV } \\
\text { POS }\end{array}$ & $\begin{array}{l}\text { HIV } \\
\text { PREVALENCE }\end{array}$ \\
\hline Jan.2005-March 2005 & 2,817 & 108 & $3.80 \%$ \\
April.2005-March 2006 & 12,076 & 675 & $5.60 \%$ \\
2006 April.-March 2007 & 26,987 & 1,314 & $4.90 \%$ \\
2007 April -2008 March & 80,117 & 3,534 & $4.40 \%$ \\
2008 April.-March 2009 & 138,683 & 5,409 & $3.90 \%$ \\
2009 April.-March 2010. & 188,675 & 5,960 & $3.20 \%$ \\
2010 April -March 2011 & 236,053 & 7,681 & $3.30 \%$ \\
2011 April.-Sept 2011 & 151,625 & 4,237 & $2.80 \%$ \\
Oct.11-Sept.2012 & 21,787 & 485 & $2.23 \%$ \\
Oct.2012-Sept.2013 & 56,531 & 1,294 & $2.29 \%$ \\
TOTAL & 915,351 & 30,697 & AVERAGE=3.35\% \\
\hline
\end{tabular}

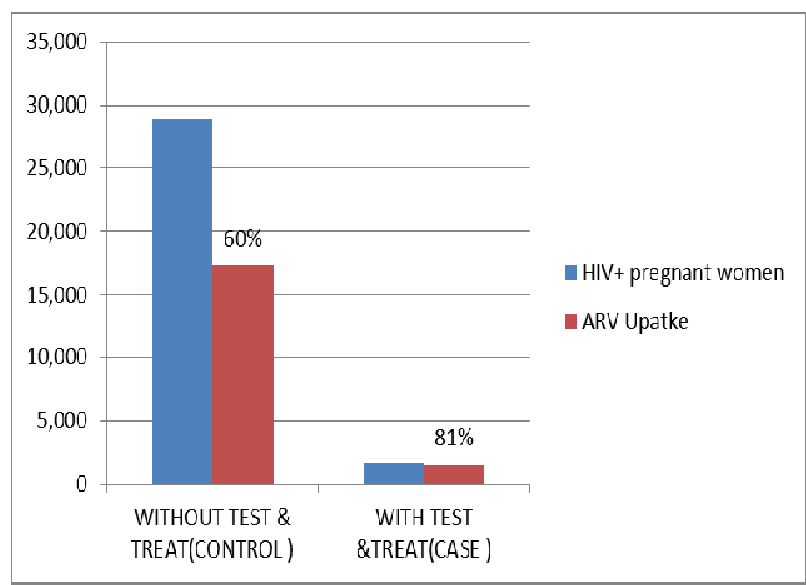

Fig 1. Comparison ARV uptake between Control and Case

Table 3. Data Summary Table

\begin{tabular}{llll}
\hline & ARV Uptake & $\begin{array}{l}\text { HIV+ Pregnant } \\
\text { Women }\end{array}$ & Total \\
\hline $\begin{array}{l}\text { With Test \& Treat } \\
\text { (Case) }\end{array}$ & 1,534 & 1,779 & 3,313 \\
$\begin{array}{l}\text { Without Test } \\
\text { \&Treat (Control) }\end{array}$ & 17,254 & 28,918 & 46,172 \\
$\begin{array}{l}\text { Total } \\
\text { Odds Ratio }\end{array}$ & 18,788 & 30,697 & 49,485 \\
$\begin{array}{l}\text { 95\% Confidence } \\
\text { Interval }\end{array}$ & 1.44 & & \\
p- value & 0.00 & & \\
\hline
\end{tabular}

Table 2. Antiretroviral Upatke Among Hiv Positive Pregnant Women

\begin{tabular}{llll}
\hline \multicolumn{1}{l}{ ARV USE AMONG HIV + ANC CLIENTS } & & \\
\hline \multicolumn{1}{l}{ Dates } & Hiv Pos Preg. Women & Number On Arv Drugs & Percentage of Arv Uptake \\
\hline & WITHOUT TEST AND TREAT STRATEGY(CONTROLS) & $10 \%$ \\
Jan.2005-March 2005 & 108 & 11 & $56 \%$ \\
April.2005-March 2006 & 675 & 381 & $49 \%$ \\
2006 April.-March 2007 & 1,314 & 640 & $54 \%$ \\
2007 April -2008 March & 3,534 & 1,916 & $67 \%$ \\
2008 April.-March 2009 & 5,409 & 3,600 & $60 \%$ \\
2009 April.-March 2010. & 5,960 & 3,592 & $60 \%$ \\
2010 April -March 2011 & 7,681 & 4,574 & $59 \%$ \\
2011 April.-Sept 2011 & 4,237 & 2,540 & Average $=60 \%$ \\
TOTAL & 28,918 & 17,254 & $69 \%$ \\
& With Test and Treat Strategy(Cases) & & $93 \%$ \\
Oct.11-Sept.2012 & 485 & 334 & Average $=81 \%($ OR $=1.4)$ \\
Oct.2012-Sept.2013 & 1,294 & 1,200 & \\
TOTAL & 1,779 & 1,534 & \\
\hline
\end{tabular}

\section{Discussion}

From table 1, a total of 915,351 pregnant women were screened for HIV in both the control and case arms using HIV antibody rapid test kit. The serial HIV testing algorithm was used as stipulated in the Nigerian national guideline (8) .Of this number 30,697 were HIV positive, divided into 28,918 controls and 1,534 cases. This figure represents $3.35 \%$
HIV prevalence among the pregnant women. This prevalence is not different from the national HIV prevalence of $4.1 \%$ as confirmed by the 2010 Nigerian National HIV seroprevalence Sentinel Survey among antenatal clients (9). In the control group, an average of $60 \%$ of the 28,918 HIVPOSITIVE pregnant women were initiated on ARV for PMTCT while among the cases $81 \%$ of the HIV positive pregnant women was placed on $\mathrm{ARV}$ with an $\mathrm{OR}=1.4$ 
$(\mathrm{CI}=1.35-1.55 \mathrm{P}=0.00)$. The calculated odds ratio of 1.4 means that an HIV positive pregnant woman has 1.4 chance of being placed on ARV in a test and treat setting than in non-test and treat situation. In other words, an HIV POSITIVE woman has $40 \%$ greater chance of being placed on ARV with test and treat strategy than without the strategy. In fact, the proportion of those ever initiated on ARV among the controls was between $10 \%$ and $67 \%$ while the fraction of those initiated on ARV in the case group range from $70 \%$ to over $90 \%$ (table 2).It is expected that the proportion of patients who are retained in care will increase as patients now have reasons to return to the clinic for their ARV refill. With excellent adherence to ARV by the HIV POSITIVE pregnant women, the maternal viral load is expected to fall to an undetectable level within few months of ART, and consequent transmission rate of HIV to the fetus and the newborn is expected to be reduced to less than $1 \%$. Limitations to this study include non-probability sampling method of selection of study participants, no matching of control and cases and small sample size of case compared with the control

\section{Conclusion}

Test and Treat Strategy is an effective way of improving ARV uptake among HIV POSITIVE pregnant women especially in resource-constraint setting where it is difficult to get the pre-requisite baseline CD4 investigations and other tests available as at when needed.

\section{Recommendations}

Immediate commencement of $\mathrm{ARV}$ (Test and Treat) in HIV POSITIVE pregnant women without waiting for CD4 test result should be encouraged and make a standard of care for PMTCT especially in resource-poor settings worldwide. Further research work on the direct impact of Test \& Treat strategy on the vertical transmission of HIV infection in children should be done

\section{Acknowledgement}

Institute of Human Virology Nigeria(IHVN)-ACTION Project.

Equitable Heath Access Initiative EHAI-ACTIONPlus-Up Project

\section{References}

[1] UNAIDS 2013 Report on the global AIDS epidemic (http://www.unaids.org/en/resources/presscentre/pressreleas eandstatementarchive/2013/september/20130923prunga/[9/2 6/2013]

[2] WHO, UNAIDS and UNICEF. Progress report 2011: global HIV/AIDS response. Epidemic uptake and health sector progress towards universal access. Geneva, World Health Organization, 2011 (www.who.int/hiv/pub/progress_report2011/en/index.html, accessed 15 May 20.

[3] Cohen MS, Chen YQ, McCauley M, et al. Prevention of HIV-1 infection with early antiretroviral therapy. N Engl J Med. 2011;365:493-505.

[4] Joint United Nations Programme on HIV/AIDS, 2013 Progress on the Global Plan towards the Elimination of New HIV Infections among Children by 2015 and Keeping their Mothers Alive, UNAIDS, Geneva, 2013.

[5] World Health Organization, The Joint United Nations Programme on HIV/AIDS, the United Nations Children's Fund. Towards universal access: scaling up priority HIV/AIDS interventions in the health sector: progress report 2008. Geneva: WHO;2008.

[6] Nigerian National Agency for the Control of AIDS: Global AIDS Response Progress report (GARPR), 2012 pg. 30

[7] Consolidated Guidelines on the Use of Antiretroviral Drugs for Treating and Preventing HIV Infection: Recommendations for a public health approach, World Health Organization, June 2013

[8] Nigerian National PMTCT Guidelines 2010, Laboratory Diagnosis of HIV Infection in Pregnant Women, pg 10-18

[9] 2010 National HIV Sero-prevalence Sentinel Survey .page 44

[10] David B. H, Nancy A. H, Elizabeth T. G. et al. Increase in Single-Tablet Regimen Use and Associated Improvements in Adherence-Related Outcomes in HIV-Infected Women J Acquir Immune Defic Syndr. 2014;65: 587-596.

[11] Carla J. C, Mark J. G, Nande P, et al. Optimal Time on HAART for Prevention of Mother-to-child Transmission of HIV . J Acquir Immune Defic Syndr. 2011;58: 224-228

[12] Federal Ministry of Health, National Scale Up Plan Towards Elimination Of Mother To Child Transmission Of HIV In Nigeria 2010 - 2015 pg.2-17. 